\title{
Contribuição da matéria autóctone e alóctone para a dieta de Bryconamericus microcephalus (Miranda-Ribeiro) (Actinopterygii, Characidae), em dois trechos de um riacho de Mata Atlântica, Rio de Janeiro, Brasil
}

\author{
Carla F. Rezende \& Rosana Mazzoni
}

Programa de Pós Graduação em Biologia, Departamento de Ecologia, Instituto de Biologia Roberto Alcântara Gomes, Universidade do Estado do Rio de Janeiro. Avenida São Francisco Xavier 524, 20550-013 Rio de Janeiro, Rio de Janeiro, Brasil. E-mail: carla.fr@terra.com.br; mazzoni@uerj.br

\begin{abstract}
Contribution of autochtonous and alochtonous matter for the diet of Bryconamericus microcephalus (Miranda-Ribeiro) (Actinopterygii, Characidae), in two streches of a Mata Atlântica stream, Rio de Janeiro, Brazil. Feeding habit of Bryconamericus microcephalus (Miranda-Ribeiro, 1908) was studied in order to detect differences related to habitat characteristics. Bimonthly samples were conducted during two annual cycles (August 2001 to September 2003), in two sites, $80 \mathrm{~m}$ long, that differed in the canopy densities (LF - covered site) and (LA uncovered site). At each sampling date and site 15 B. microcephalus specimens were sampled. Stomach content of each specimen was analyzed through Numeric Frequency and Frequency of Occurrence. Food items were, then, classified as autochthonous or allochthonous, according to their origin. Insects were the main food item consumed by the specimens from both sites. Considering the analysis of LF no significant differences were registered for the consumption of allochthonous e autochthonous items. Nonetheless, predominance of autochthonous preys was detected in LA. Seasonal and spatial analyses of the contribution of allochthonous and autochthonous items, during the first study year, indicated that autochthonous prey prevailed during the dry season of LF and the rainy season of LA; no significant differences were registered during the second studied year. It is concluded that feeding habit of B. microcephalus did not varied in a predictable pattern.

KEY WORDS. Canopy; feeding resource; llha Grande; seasonality.
\end{abstract}

RESUMO. O hábito alimentar de Bryconamericus microcephalus (Miranda-Ribeiro, 1908) foi analisado com o objetivo de se avaliar suas diferenças relacionadas às características ambientais. Coletas bimestrais foram realizadas durante dois ciclos anuais (Agosto/2001 a Setembro/2003), em duas localidades, $80 \mathrm{~m}$ de extensão, que diferem pela densidade da cobertura vegetal (LF - localidade fechada) e (LA - localidade aberta). Em cada ocasião de coleta e localidade foram amostrados 15 exemplares de B. microcephalus. O conteúdo estomacal de cada exemplar foi analisado pelos métodos da Freqüência Numérica e Freqüência de Ocorrência. Os itens alimentares foram classificados como autóctone ou alóctone, de acordo com sua origem. Os insetos foram o principal item alimentar consumido pelos indivíduos de ambas as localidades. Considerando-se a análise de LF não foram registradas diferenças no consumo dos itens alóctones e autóctone. Por outro lado, foi detectada predominância de presas autóctones em LA. A análise espaço-temporal da participação dos itens alóctones e autóctones, durante o primeiro ano de estudo, indicaram que as presas autóctones predominaram na estação seca de LF e na estação chuvosa de LA; não foram registradas diferenças significativas durante o segundo ano de estudo. Conclui-se que as variações espaço-temporais do hábito alimentar de $B$. microcephalus não seguiram um padrão previsível.

PALAVRAS CHAVE. Cobertura vegetal; Ilha Grande; recurso alimentar; sazonalidade.

Estudos sobre alimentação de peixes vêm se tornando cada vez mais numerosos devido à necessidade de dados para a formulação de modelos sobre a estrutura trófica de ecossistemas, além do entendimento dos mecanismos biológicos de interações interespecíficas, tais como predação e competição (ZAVALA-CAMIN 1996).
Considerando que as espécies de peixes respondem às mudanças ambientais em diferentes escalas (Rosemberger \& ANGERMEIER 2003), estudos sobre o comportamento alimentar de populações de peixes em diferentes ambientes vêm sendo desenvolvidos a fim de se esclarecer as diferenças na composição da dieta de populações distintas (Mittelbach 1981, AbujanRA 
et al. 1999, Harrel \& Diblle 2001, Dufech et al. 2003).

Os diferentes tipos de ambiente utilizados por uma população podem estar diretamente relacionados com o aporte de energia (Mittelbach 1981, Garman 1991). No caso de ambientes de riachos, a matéria orgânica particulada e a matéria de origem terrestre possuem grande contribuição como fonte de nutrientes (VANNOTE et al. 1980) podendo, desta forma, influenciar o comportamento dos peixes (GARMAN 1991).

Estudos recentes sobre a alimentação de peixes têm demonstrado que várias espécies de Characidae dependem de recursos alimentares derivados das matas ciliares (LoweMcConnell 1987, Dufech et al. 2003, Graciolli et al. 2003, ReZende \& Mazzoni 2003) e dessa forma, mudanças na composição e na estrutura da vegetação podem causar alterações na disponibilidade de alimento (ANGermeIER \& KarR 1983) e, conseqüentemente, nos hábitos alimentares dos peixes (Dufech et al. 2003).

Neste trabalho são apresentados resultados relativos à dieta do lambari Bryconamericus microcephalus do córrego Andorinha, considerando-se duas localidades com diferentes quantidades de cobertura vegetal durante duas estações: seca e chuva. Espera-se que a dieta dos indivíduos da localidade sem cobertura vegetal apresente maior incidência de itens de origem autóctone enquanto os indivíduos da localidade com cobertura apresentem os itens alóctones como sua principal fonte de alimento e que a contribuição dos itens de origem alóctone seja maior nas duas localidades durante a estação de chuva.

\section{MATERIAL E MÉTODOS}

A Ilha Grande $\left(23^{\circ} 11^{\prime} 11,7^{\prime \prime} S ; 44^{\circ} 12^{\prime} 02,0^{\prime \prime} \mathrm{W}\right)$ está localizada na costa sul do Estado do Rio de Janeiro e é composta por floresta densa ombrófila de Mata Atlântica primária e/ou secundária (AlHo et al. 2002). O córrego Andorinha, local do presente trabalho, é um riacho de terceira ordem que drena a vertente oriental da Ilha e percorre uma extensão aproximada de $10 \mathrm{~km}$, desde sua nascente até a desembocadura na Enseada dos Dois Rios. Ao longo do seu percurso, apresenta grande variação de relevo, intercalando áreas de corredeiras, rápidos e remansos e percurso subterrâneo em alguns trechos.

Ao longo do eixo longitudinal do córrego Andorinha foram selecionadas duas localidades de estudo que se distinguem pela altitude e pelo grau de cobertura vegetal. A primeira localidade, denominada "localidade fechada" (LF), está situada a $150 \mathrm{~m}$ a.s.l. e apresenta aproximadamente 95\% de cobertura vegetal composta por Mata Pluvial Atlântica secundária. A segunda localidade, denominada "localidade aberta" (LA), está situada a $50 \mathrm{~m}$ a.s.l., não apresentando cobertura vegetal e a vegetação ripária se caracteriza por pequenos arbustos e gramíneas marginais ao leito do riacho. Ambas as localidades apresentavam características hídricas semelhantes, com predomínio de rápidos, intercalados por remansos marginais e substrato rochoso intercalado por poços areno-lodosos e abundante matéria orgânica depositada.

Em cada localidade foi amostrado um trecho de aproxi- madamente $80 \mathrm{~m}$ de extensão. As coletas foram realizadas com arrastinhos (malha 0,5 mm), redes de espera (malha $10 \mathrm{~mm}$ ), peneiras e puçás. As redes de espera eram expostas das 13:00 as 15:30 h e vistoriadas a intervalos de 30 minutos. Paralelamente à exposição das redes de espera, nas áreas marginais da localidade e fora da área de exposição das redes, foram utilizados arrastos manuais e peneiras na intenção de se amostrar a maior variabilidade de tamanho dos exemplares e microhábitats utilizados pela espécie. Dessa forma, a cada ocasião de amostragem, foram coletados aproximadamente 15 exemplares de tamanhos variados.

As amostragens foram realizadas bimestralmente entre agosto de 2001 e setembro de 2003 e compreenderam dois ciclos de doze meses (Ciclo I - agosto, outubro, dezembro de 2001; fevereiro, abril e junho de 2002; e Ciclo II - outubro, dezembro de 2002; fevereiro, maio, julho e setembro de 2003). Como os dois ciclos diferem quanto ao número de meses chuvosos e secos o total de indivíduos analisados variou entre as duas estações.

Considerando que a dieta da espécie é composta principalmente por artrópodos terrestres e insetos e larvas aquáticas existentes no substrato (Rezende \& Mazzoni 2003) e que o regime de chuvas na região neotropical é o principal fator que age sobre estrutura do substrato alterando a dinâmica de seus recursos tróficos em diversos rios e riachos tropicais (e.g. LoBónCerviá \& Bennemann 2000, Mazzoni \& Lobón-Cerviá 2000), as análises do presente estudo foram realizadas com base em duas estações do ciclo anual: (I) estação seca e (II) estação chuvosa.

Para a distinção das estações de seca e chuva tomamos por base a afirmação de SOARES (2000) de que somente chuvas iguais ou superiores a 25,2 mm afetam o caudal dos rios. Desta forma, foram considerados como meses de chuva aqueles que apresentaram chuvas igual ou superior a 25,2 $\mathrm{mm}$ em até 10 dias antes da coleta de dados. Para o ciclo I foram considerados dezembro/2001 e fevereiro, abril, e junho/2002 como meses de chuva e os demais como meses de seca. Para o ciclo II foram considerados como meses de chuva dezembro/2002 e maio/ 2003 e os demais como meses de seca (Fig. 1).

A cada ocasião de coleta os exemplares amostrados eram mantidos em gelo e transportados ao laboratório para processamento. Para cada exemplar foram registrados os dados de: comprimento padrão ( $\mathrm{CP}$ - centímetros, aproximação de milímetros), peso total (PT - gramas, aproximação de miligramas) e comprimento do intestino (CI - centímetros, aproximação de milímetros). O estômago de cada exemplar foi removido, pesado (PE - gramas, aproximação miligramas) e fixado em formol $5 \%$ para posterior análise.

O conteúdo dos estômagos foi analisado sob microscópio estereoscópico e microscópio óptico, de acordo com os métodos da Freqüência Numérica e Freqüência de Ocorrência (Hyslop 1980). Para efeito de análise dos dados, os itens alimentares registrados foram agrupados de acordo com sua origem, tendo-se, portanto, duas categorias de itens: autóctone e

Revista Brasileira de Zoologia 23 (1): 58-63, março 2006 


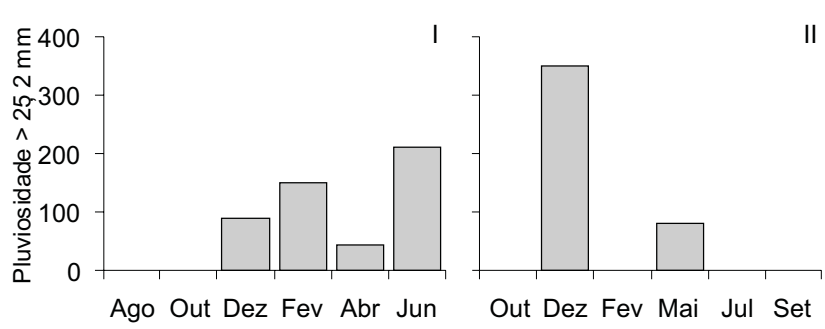

Figura 1. Somatório mensal das precipitações, superiores a 25,2 $\mathrm{mm}$, que ocorreram até 10 dias antes da amostragem nos ciclos I (I) e ciclo II (II). Fonte de dados Central Nuclear Almirante Álvaro Alberto, Angra dos Reis, Rio de Janeiro.

alóctone. A participação relativa de cada categoria foi calculada para cada área de estudo e para as estações de seca e chuva. Com base na hipótese de que a abundância de matéria alóctone e autóctone independe da localidade amostral considerada, aplicou-se o teste do Qui-Quadrado $\left(\chi^{2}\right)$ de contigência 2 × 2 (ZAR 1999).

A fim de se estabelecer o grau de especificidade da dieta da espécie realizou-se a análise gráfica (Costello 1990) que associa os métodos de Freqüência de Ocorrência e Freqüência Numérica.

\section{RESULTADOS}

Foram coletados 371 indivíduos, sendo 32 na estação seca e 51 na estação chuvosa no ciclo I e 79 na estação seca e 30 na estação chuvosa no ciclo II, para a localidade fechada. Na localidade aberta foram coletados 31 exemplares na estação seca e 53 na estação chuvosa no ciclo I e 70 na estação seca e 28 na estação chuvosa no ciclo II.

Dentre os itens alimentares que compõe a dieta de $B$. microcephalus (Tab. I) observa-se que os artrópodes, representados em maior freqüência numérica por larvas e/ou formas adultas de insetos (terrestres e aquáticos), representam, em média, $90 \%$ dos itens consumidos. Os demais itens perfazem, reunidos, uma média de $10 \%$ da dieta e têm, normalmente, ocorrência pontual e/ou ocasional.

A análise da riqueza de tipos de presas consumidas em cada localidade e estação indica pouca variação, tanto especial como sazonal (Fig. 2), sugerindo que as variações da alimentação são qualitativas.

Através do índice gráfico de Costello (1990) apresentado na figura 3 observamos que as presas dominantes na dieta de B. microcephalus, tanto para a localidade fechada quanto para a aberta, são as larvas de insetos (localidade fechada - FN\% 49,8 e FO\% 46,98) e (localidade aberta - FN\% 68,6 e FO\% 58,3) e os insetos terrestres (localidade fechada-FN\% 26,1 e FO\% 57,8) e (localidade aberta - FN\% 22,1 e FO\% 55,95). Os demais itens são acessórios, fato comum para ambas as localidades. Com base nessa análise classificamos a espécie como insetívora.

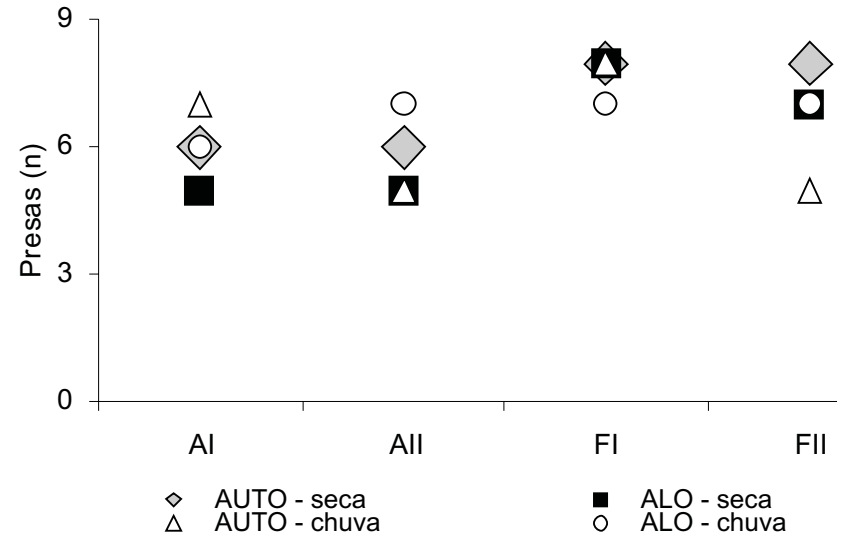

Figura 2. Variação espaço-temporal do número de presas consumidas por Bryconamericus microcephalus do córrego Andorinha. (AI) Localidade aberta no ciclo I, (AII) localidade aberta no ciclo II, (FI) localidade fechada no ciclo I, (FII) localidade fechada no ciclo II.

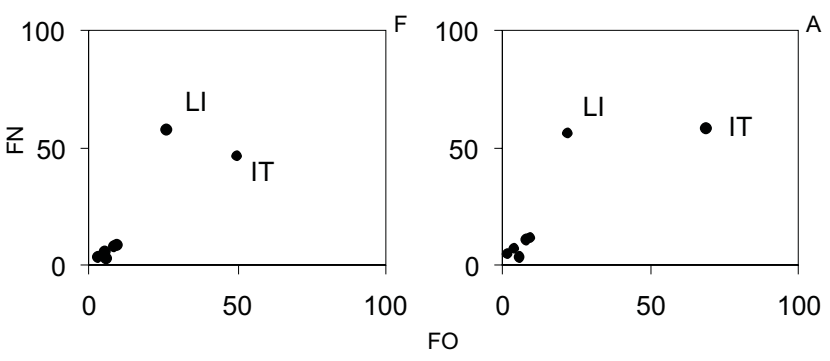

Figura 3. Caracterização da dieta de Bryconamericus microcephalus no córrego Andorinha através do índice Gráfico de Costello. (LI) Larvas de insetos, (IT) insetos terrestres, (F) localidade Fechada e (A) localidade Aberta.

Comparando a contribuição das presas autóctone e alóctone na dieta dos exemplares, em cada ciclo anual e localidade de estudo (Fig. 4), observamos que em LF não ocorrem diferenças na participação relativa de ambos os tipos de recursos, para os dois ciclos (Ciclo I $-\chi^{2}=0,38, \mathrm{p}=0,54$; Ciclo II $-\chi^{2}$ $=0,07, \mathrm{p}>0,79)$. Por outro lado, em LA evidencia-se predomínio significativo das presas autóctones no ciclo I $\left(\chi^{2}=10,05, p\right.$ $<0,001)$. No ciclo II, embora as diferenças não sejam significativas $\left(\chi^{2}=1,57, p=0,20\right)$, observa-se a mesma tendência do ciclo I. Com base, ainda, no observado na figura 4 , tem-se que a participação dos itens alóctones, presentes na dieta de $B$. microcephalus, não supera em nenhuma localidade ou ciclo estudado a participação dos itens autóctones.

A análise espaço-temporal da contribuição das presas alóctone e autóctone (Fig. 5) durante o ciclo I, indica que as presas autóctones predominaram significativamente na estação seca de LF $\left(\chi^{2}=10,27, p<0,01\right)$ e na estação chuvosa de LA 


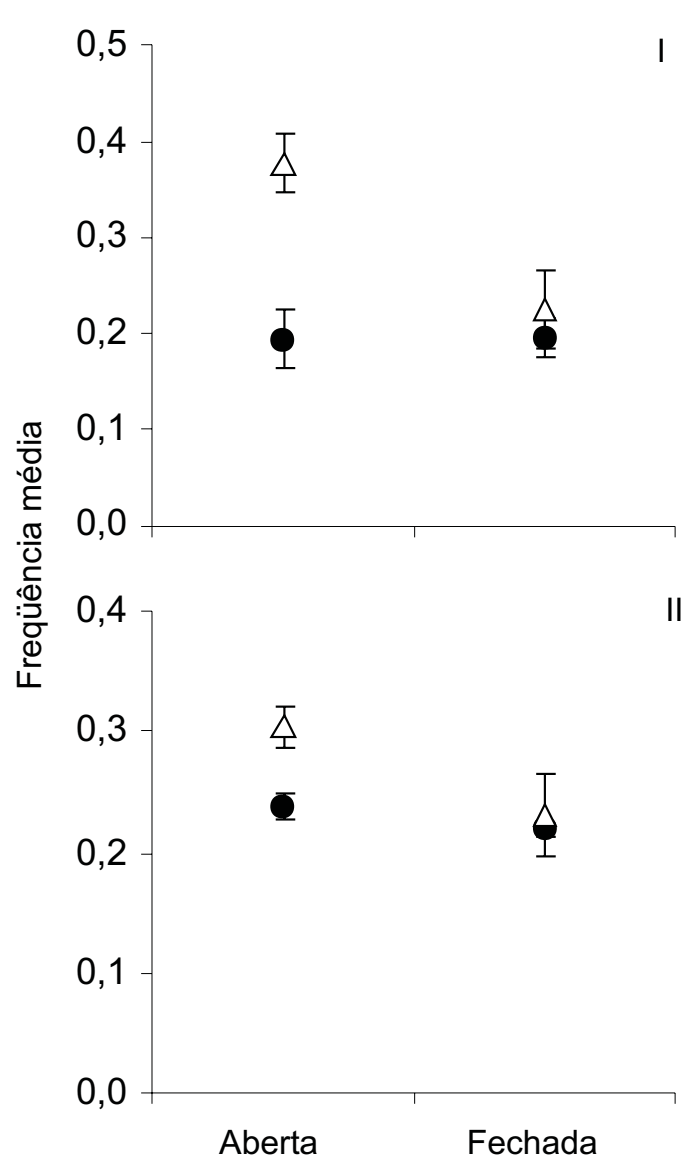

Figura 4. Valores médios da freqüência numérica dos itens alimentares de origem alóctone (círculo) e autóctone (triângulo), nas localidades Aberta e Fechada durante o ciclo I (I) e o ciclo II (II).

$\left(\chi^{2}=7,56, p<0,01\right)$. No entanto, nas estações seca e chuvosa de LA e LF, respectivamente, não ocorreram diferenças significativas $\left(\chi^{2}=1,23, p=0,27\right.$ e $\chi^{2}=1,08, p=0,29$, respectivamente). Durante o ciclo II (Fig. 5) observou-se padrão distinto do ocorrido no ciclo I; o consumo dos dois tipos de presas diferiu significativamente entre localidades e estações $(p>0,01)$.

\section{DISCUSSÃO}

Bryconamericus microcephalus, do córrego Andorinha, foi classificado como espécie insetívora sendo que a estratégia de consumo das presas varia entre estações e localidades. Sua dieta é composta basicamente por insetos, sendo os demais itens consumidos ocasionalmente. Esse resultado corrobora observações anteriores (Rezende \& MAZzoni 2003).

Efeitos da sazonalidade têm sido constatados em diversas comunidades de peixes de riachos (LitTle et al. 1998, Deus \& Petrere-Junior 2003). Para família Characidae são registrados diversos trabalhos que indicam variações sazonais na dieta das

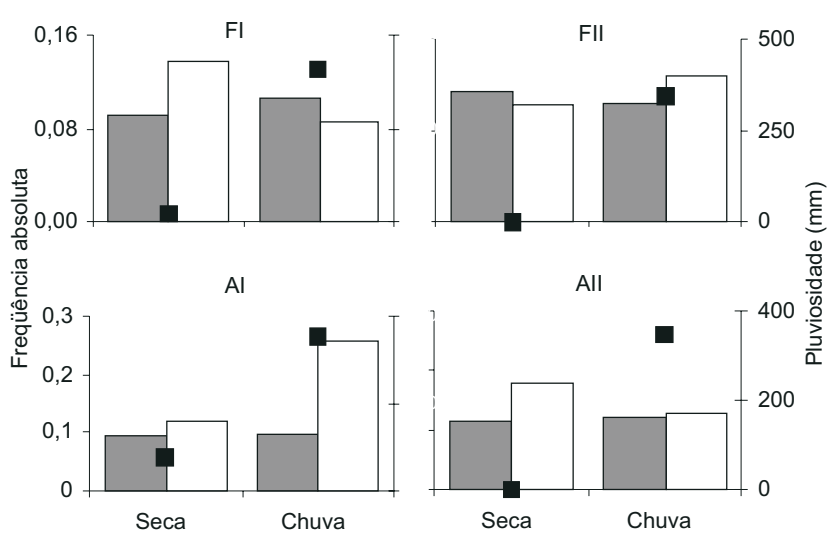

Figura 5. Comparação entre os índices pluviométricos sazonais (quadrado em preto é a soma do total de chuvas acima de 25,2 $\mathrm{mm}$ ) e a freqüência absoluta dos itens alóctone (cinza) e autóctone (branco) na dieta de Bryconamericus microcephalus, nas localidades Aberta (Al) e Fechada ( FI) do ciclo I e localidades Aberta (AII) e Fechada (FII) do ciclo II.

espécies (e.g. Aranha et al. 2000, Esteves \& Pinto-Lobo 2001, MAZZONI \& REZENDE 2003) sendo em alguns casos detectadas variações na utilização de presas preferenciais de acordo com a disponibilidade no ambiente (Deus \& Petrere-Junior 2003). Porém para $B$. microcephalus não detectamos variações sazonais no tipo de presas que compuseram a dieta da espécie.

O hábito de consumir insetos como principal recurso alimentar é amplamente estabelecido entre espécies de peixes de riachos. Muitas dessas espécies têm distribuição restrita às áreas com cobertura vegetal, pois sugere-se que nessas localidades o aporte de matéria alóctone, principalmente insetos terrestres, é superior ao registrado nas áreas sem cobertura vegetal (Welcome 1985, Luiz et al. 1998, Sabino \& Castro 1990, Mazzoni \& Iglesias-Rios 2002). A distribuição de B. microcephalus não mostrou nenhum padrão de distribuição relacionado ao grau de cobertura, uma vez que em ambas localidades estudadas a espécie era abundante.

Dufech et al. (2003), comparando duas populações de Mimagoniates rhoecharis Menezes \& Weitzman, 1990 que ocorriam em localidades diferenciadas pelo grau de cobertura vegetal, registraram diferenças significativas na participação de itens alóctones e autóctones na dieta, sendo que na localidade de mata fechada predominavam itens alóctones. O padrão alimentar registrado para B. microcephalus não corroborou essa observação. Em nenhum dos dois ciclos estudados registrou-se predomínio de itens alóctones em LF.

A importância da vegetação marginal como fonte de insetos terrestres para a alimentação de peixes de riacho foi ressaltada por ANGERMEIER \& KARR (1983) e GARMAN (1991). Da mesma forma, Miтtelbach (1981) sugere que o ganho energético das espécies de peixes difere entre áreas com e sem cobertura e que, normalmente ocorre migração de peixes para o ambiente 
Tabela I. Freqüência percentual dos itens alimentares que compõem a dieta de Bryconamericus microcephalus do córrego Andorinha, llha Grande, durante os dois ciclos temporais.

\begin{tabular}{|c|c|c|c|c|c|c|c|c|}
\hline \multirow{3}{*}{ Itens alimentares } & \multicolumn{4}{|c|}{ Ciclo I } & \multicolumn{4}{|c|}{ Ciclo II } \\
\hline & \multicolumn{2}{|c|}{$\mathrm{LF}$} & \multicolumn{2}{|c|}{ LA } & \multicolumn{2}{|c|}{ LF } & \multicolumn{2}{|c|}{ LA } \\
\hline & Chuva & Seca & Chuva & Seca & Chuva & Seca & Chuva & Seca \\
\hline \multicolumn{9}{|l|}{ Matéria Alóctone } \\
\hline \multicolumn{9}{|l|}{ Insetos Terrestres } \\
\hline Coleoptera & 5,8 & 7,1 & 4,6 & 11,6 & 6,9 & 10,40 & 6,70 & 6,6 \\
\hline Hymenoptera & 23,3 & 17,1 & 10,9 & 21,7 & 18,1 & 15,00 & 28,40 & 13,1 \\
\hline Homoptera & 2,9 & 5,7 & 2,3 & 0 & 2,8 & 2,90 & 2,50 & 3,0 \\
\hline Diptera & 5,8 & 2,9 & 9,7 & 2,9 & 2,8 & 6,40 & 1,20 & 7,7 \\
\hline Lepidoptera & 1,0 & 0 & 0 & 0 & 0 & 0 & 0 & 0 \\
\hline Orthoptera & 0 & 4,3 & 0,6 & 0 & 0 & 1,70 & 0 & 1,8 \\
\hline \multicolumn{9}{|l|}{ Arachinida } \\
\hline Acari & 1,0 & 0 & 0 & 1,5 & 0 & 0,60 & 0 & 0 \\
\hline Araneae & 1,9 & 2,9 & 0,6 & 1,5 & 0 & 2,90 & 2,50 & 1,8 \\
\hline Semente & 1,94 & 0 & 0 & 0 & 0 & 9,25 & 4,94 & 0 \\
\hline \multicolumn{9}{|l|}{ Matéria Autóctone } \\
\hline \multicolumn{9}{|l|}{ Insetos Aquáticos } \\
\hline Hemiptera & 0 & 8,6 & 1,7 & 0 & 1,4 & 2,30 & 0 & 0,6 \\
\hline Trichoptera & 1,9 & 0 & 2,3 & 0 & 0 & 0 & 0 & 0,6 \\
\hline Plecoptera & 1,0 & 1,4 & 0,6 & 1,5 & 0 & 0 & 0 & 0 \\
\hline \multicolumn{9}{|l|}{ Larva de insetos } \\
\hline Larva de Coleoptera & 1,0 & 4,3 & 0 & 0 & 2,8 & 1,20 & 1,20 & 2,4 \\
\hline Larva de Chiromidae & 36,9 & 34,3 & 25,1 & 31,0 & 50,0 & 27,20 & 16,10 & 26,8 \\
\hline Pupa de Chironomidae & 4,9 & 1,4 & 36,0 & 27,5 & 11,1 & 17,30 & 34,60 & 33,9 \\
\hline Larva de Odonata & 3,9 & 7,1 & 4,0 & 1,5 & 0 & 0,60 & 1,20 & 1,2 \\
\hline Ephemeroptera & 3,9 & 0 & 0,6 & 0 & 2,8 & 2,30 & 0 & 0,6 \\
\hline \multicolumn{9}{|l|}{ Crustáceos } \\
\hline Decapoda & 1,0 & 1,4 & 0 & 0 & 1,4 & 0 & 0 & 0 \\
\hline Caranguejo & 1,9 & 0 & 0,6 & 0 & 0 & 0 & 0 & 0 \\
\hline Copepoda & 0 & 0 & 0 & 0 & 0 & 0 & 1,20 & 0 \\
\hline
\end{tabular}

com maior disponibilidade de recursos. No caso, de B. microcephalus a população era abundante em LA e LF e a maioria dos estômagos encontravam-se com alto grau de repleção nas duas localidades o que pode ser um indício de grande disponibilidade de recurso nos dois ambientes.

Os resultados aqui apresentados sugerem que a dieta de $B$. microcephalus no Córrego Andorinha apresentou pouca alteração espaço-temporal e que os padrões encontrados não se repetiram durante os dois ciclos estudados. Por outro lado, é importante ressaltar que durante os dois anos de coleta o regime de chuvas da região variou de forma aleatória o que pode ter ocasionado a ausência de um padrão nos resultados aqui apresentados. Ainda nesse contexto há possibilidade dos indivíduos das duas localidades estarem refletindo um padrão temporal da disponibilidade de recursos que não foi avaliado neste trabalho.

\section{AGRADECIMENTOS}

Este trabalho é parte da dissertação de mestrado da primeira autora (CFR) e foi financiado pelo projeto CNPq (APQ2002-479426/01-5). Agradecemos as bolsas individuais recebidas pelas autoras CFR, FAPERJ, E-26/151.406/2003 e RM - CNPq - 302628/2002-9 e a todos os membros do Laboratório de Ecologia de Peixes, UERJ pela ajuda nos trabalhos de campo.

\section{REFERÊNCIAS BIBLIOGRÁFICAS}

Abujanra, F.; M.R. Russo \& N.S. Hahn. 1999. Variações espaçotemporais na alimentação de Pimelodus ortamanni (Siluriformes, Pimelodidae) no reservatório de Segredo e áreas adjancetes (PR). Acta Scientiarum, Maringá, 21 (2): 283-289. Alho, C.J.R.; M. Schneider. \& L.A. Vasconcellos. 2002. Degree of 
the threat to the biological diversity in the Ilha Grande state park (RJ) and the guidelines for conservation. Brazilian Journal of Biology, São Carlos, 62 (3): 375-385.

Angermeier, P.L. \& J.R. Karr. 1983. Fish communities along environmental gradients in a system of tropical streams. Environmental Biology of fishes, Dordrecht, 9 (2): 117-135.

Aranha, M.R.; J.H.C. Gomes \& F.N. Fogaça. 2000. Feeding of two sympatric species of Characidium, C. lanei and C. pterostictum (Characidiinae) in a coastal stream of Atlantic Forest (Southern Brazil). Brazilian Archives of Biology and Technology, Curitiba, 43 (5): 527-531.

Costello, M.J. 1990. Predator feeding strategy and prey importance: a new graphical analysis. Journal of Fish Biology, London, 36: 261-263.

Deus, C.P. \& M. Petrere-Junior. 2003. Seasonal diet shifts on seven fish species in an Atlantic Rainforest stream in southeastern Brazil. Brazilian Journal of Biology, São Carlos, 63 (4): 579 588.

Dufech, A.P.S.; M.A. Azevedo \& C.B. Fialho. 2003. Comparative dietary analysis of two populations of Mimagoniates rhocharis (Characidae: Glandulocaudinae) from two streams of Southern Brazil. Neotropical Ichthyology, Porto Alegre, 1 (1): 67-74.

Esteves, K.E. \& A.V. Pinto-Lobo. 2001. Feeding Pattern of Salminus maxillosus (Pisces, Characidae) at Cachoeira das emas, MogiGuaçu River (São Paulo State, Southerneast Brazil). Brazilian Journal of Biology, São Carlos, 61 (2): 267-276.

GARMAN, G.C. 1991. Use of terrestrial arthropod prey by a streamdwelling cyprinid fish. Environmental Biology of Fishes, Dordrecht, 30: 325-331.

Graciolli, G.M.; A. Azevedo \& F.A.G. Melo. 2003. Comparative study of the diet of Glandulocaudine and Tetragonopterinae (Ostariophysi: Characidae) in a small stream in southern Brazil. Studies on Neotropical Fauna and Environment, Lisse, 38 (2): 95-110.

Harrel, S.L. \& E.R. Dibble. 2001. Foraging efficiency of juvenile bluegill, Lepomis macrochirus, among different vegetated habitats. Environmental Biology of Fishes, Dordrecht, 62: 441-453.

Hyslop, E.J. 1980. Stomach contents analysis - a review of methods and their application. Journal of Fish Biology, London, 17: 411-429.

Little, A.S.; W.M. Tonn; R.F. Tallman \& J.D. Reist. 1998. Seasonal variation in diet and trophic relationships within the fish communities of the lower Slaver River, Northwest Territories, Canada. Environmental Biology of Fishes, Dordrecht, 53: 429-445.

Lobón-Cerviá, J. \& S. Bennemann. 2000. Temporal trophic shifts and feeding diversity in two sympatric, neotropical, omnivo- rous fishes: Astyanax bimaculatus and Pimelodus maculatus in Rio Tibagi (Paraná, Southern Brazil). Archiv fur Hydrobiologie, Stuttgart, 149: 205-306.

LOWE-McConNeLL, R.H. 1987. Ecological studies in tropical fish communities. Cambrige, Cambrige University Press, 382p.

Luiz, E.A.; A.A. Agostinho; L.C. Gomes \& N.S. Hahn. 1998. Ecologia trófica de peixes em dois riachos da bacia do rio Paraná. Revista Brasileira de Biologia, São Carlos, 58: 273-285.

Mazzoni, R. \& J. Lobón-Cerviá. 2000. Longitudinal structure, density and production rates of a neotropical stream fish assemblage: the river Ubatiba in the Serra do Mar, southeast Brazil. Ecography, Copenhagen, 23: 588-602.

Mazzoni, R. \& R. Iglesias-Rios. 2002. Distribution pattern of two fish species in a coastal stream in southeast Brazil. Brazilian Journal of Biology, São Carlos, 62 (1): 171-178.

Mazzoni, R. \& C.F. Rezende. 2003. Seasonal diet shift in a Tetragonopterinae (Osteichthyes, Characidea) from Ubatiba river, RJ, Brazil. Brazilian Journal of Biology, São Carlos, 63 (1): 69-74.

Mittelbach, G.C. 1981. Foraging efficient and body size: a study of optimal diet and habitat use by bluegills. Ecology, Arizona, 62: 1370-1386.

Rezende, C.F. \& R. Mazzoni. 2003. Aspectos da alimentação de Bryconamericus microcephalus (Characiformes, Tetragonopterinae) no Córrego Andorinha, Ilha Grande - RJ. Biota Neotropica, Campinas, 3 (1): 1-6.

Rosemberger, A. \& P.L. Angermeier. 2003. Ontogenetic shifts in habitat use by the endangered Roanoke logperch (Percina rex). Freshwater Biology, Oxford, 48: 1536-1577.

SABINO, J. \& M.C. CASTro. 1990. Alimentação, período de atividade e distribuição espacial dos peixes de um riacho da Floresta Atlântica (sudeste do Brasil). Revista Brasileira de Biologia, São Carlos, 50 (1): 23-36.

SoARES, J.V. 2000. Curso de hidrologia de florestas. Instituto Nacional de Pesquisas Espaciais. Disponível na World Wide Web em: http://www.Itid.inpe.br/dsr/vianei/CursoHidFlo. html [acesso em: 10/XII/2004].

Vannote, R.L.; G.W. Minshall; K.W. Cummins; J.R. Sedell \& C.E. CuSHING 1980. The river continuum concept. Canadian Journal of Fisheries And Aquatic Sciences, Ottawa, 37: 130-137.

Welcomme, R.L. 1985. Ontogenetic diet shifts and resource partitioning among piscivorous fishes in the Venezuelann Illanos. Environmental Biology of Fishes, Dordrecht, 26: 177-199.

ZAR, J.H. 1999. Biostatistical analysis. New Jersey, PrenticeHall, $4^{\text {th }}$ ed., XII+663p.

ZaVALA-CAMIN, L.A. 1996. Introdução ao estudo sobre alimentação natural em peixes. Maringá, Editora da Universidade Estadual de Maringá, 129p.

Recebido em 06.V.2005; aceito em 22.II.2006.

Revista Brasileira de Zoologia 23 (1): 58-63, março 2006 